\title{
Relationship between autism and mind perception of selves, others, and toy objects
}

\author{
Rachel Hoi Yan $\mathrm{Au}$ \\ Department of Psychology, The Chinese University of Hong Kong
}

\begin{abstract}
Autism is found to be associated with mind perception, in particular reduced perception of human agency. Prior work has shown that more autistic individuals in the general population tend to view others as less capable of doing and thinking. Autism is, by contrast, not meaningfully associated with the mind attribution to other types of entities examined in past work, including children, nonhuman animals, supernatural agents, and so forth. In this paper, more types of entities-toy objects and selves-were investigated. Across two normal adult samples and a meta-analysis, autism was found to be associated with decreased perception of both experiential and agentic mental capacity in other adult humans, as well as in oneself. More autistic individuals tend to consider others and themselves less capable of both doing and feeling. Autism was also associated with increased mind perception of toy objects, such that more autistic individuals tend to consider toy objects more capable of doing and feeling. These findings partially replicated and meaningfully extended previous work on autism and mind perception, thus providing insight into the relationship of autism with potentially different perceptions and treatments of others, selves, and toy objects.
\end{abstract}

Keywords: Autism Spectrum Quotient, autism, autism spectrum conditions, mind perception, mind attribution

Autism is most commonly known as a clinical disorder, characterized by what is called the Autistic Spectrum Condition (ASC; DSM-V; American Psychiatric Association, 2013), expressed in poor social and communication skills, poor imagination, a strong focus of attention, phenomenal attention to details, and restricted and repetitive behavioral patterns (Baron-Cohen et al., 2001). Recent research has shown that autism is not only a condition of a specific group of individuals, but that it grades on a scale from psychopathology to normality (Ford et al., 2017). According to Baron-Cohen et al. (2001) and Constantino and Todd (2003), individuals of the normal population can also be differentiated in terms of being autistic to a certain degree. The present work investigated the role of autism as a personality disposition in relation to mind perception - the view of who and what possess mental capacities (Wegner \& Gray, 2016).

Autism has important implications on mind perception. Mind is described in terms of mental capacities to feel, think, and act. According to Gray, Gray, and Wegner (2007), these mental capacities can be understood along two dimensions - agency and experience. Agency is the capacity for doing and thinking, while experience is the capacity for feeling and sensation. Despite discussion on the preferred number of dimensions of mind perception and their specific contents (e.g., Malle, 2019), the current work is executed along the two dimensions proposed by Gray et al. (2007), because of the amount of research that has been

Correspondence concerning this article should be addressed to Rachel Hoi Yan Au, Department of Psychology, New Mexico State University, Las Cruces, NM, USA. E-mail: ahyrachel@link.cuhk.edu.hk done assuming those dimensions (e.g., Cooley et al., 2017; Doyle \& Gray, 2020; Gray, Knobe et al., 2011).

People in general tend to attribute relatively high levels of both experience and agency to human adults, relatively high levels of experience but relatively little agency to human babies and nonhuman animals, relatively little experience but relatively high levels of agency to entities of religious beings and robots, and little or no experience and agency to objects and dead entities (Gray et al., 2007; Wegner \& Gray, 2016).

There are individual differences in mind perception patterns that can be partially explained by personality traits as measured by the Big Five (e.g., John \& Srivastava, 1999). Big Five Agreeableness, for example, was found to be related to a higher level of agency perception, but not to experience perception (Tharp et al., 2017). Autism as a personality disposition, has also been found to relate to mind perception (Gray, Adrianna et al., 2011). In a normal adult sample, a small-sized but statistically significant negative correlation between autism and human agency perception was found, suggesting that individuals scoring higher on autism tend to ascribe less agentic mental capacity, such as planning and self-control, to other adults. Autism was, however, not significantly associated with experience perception in human adults nor, more generally, with mind perception in other entities. Tharp and colleagues (2017) later replicated the findings by Gray, Adrianna et al. (2011) on the relationship between autism and mind perception of various entities (e.g., baby, man, robot, tree).

In the present work, the first aim was to replicate the linkage between autism and reduced perception of human agency as found in Gray, Adrianna et al. (2011) and Tharp et al. (2017). The second aim was to extend past work by 
examining more types of entities. The mind perception targets being studied in previous research were mainly adult humans, human infants, deceased humans, nonhuman animals, supernatural agents, superheroes, robots, and trees (Gray, Adrianna et al., 2011; Tharp et al., 2017). In the current research, two other types of entities were added, namely toy objects and selves.

\section{Toy objects as targets}

Because of their difficulty in understanding other people (Michaud \& Théberge-Turmel, 2002) and their lack of motivation to socially engage with others (Chevallier et al., 2012), autistic individuals tend to experience loneliness (Jobe \& White, 2007; Mazurek, 2014). However, not only other people can serve as sources of social connection. In general, when others are unavailable, people may turn to nonhuman entities (Epley, Waytz et al., 2008), such as animal pets and religious beings (Epley, Akalis et al., 2008; Epley, Waytz et al., 2008). They usually ascribe mental capacities to these entities in order to socially connect with them (Waytz et al., 2010c). Toys form a potential type of entities that individuals high on autism are likely to perceive as having mental capabilities.

When it comes to selecting a source of social connection, more autistic individuals may be likely to choose toy objects, because of the high predictability of the actions of toys. Autism is associated with strong preferences for predictability and sameness (Baron-Cohen, 2002; Baron-Cohen et al., 2003; Goris et al., 2020; Michaud \& ThébergeTurmel, 2002; Van de Cruys et al., 2014). Thus, autistic individuals may like to turn to toy objects as a source of social interaction, and this may in turn lead them to attribute more mind to toys (e.g., Gardner \& Knowles, 2008; Kozak et al., 2006). Some recent work seems to support this possibility. Atherton and Cross (2018) have reviewed the empirical evidence for greater social interests of autistic individuals in objects than in humans. Autistic individuals were found to be inclined to perceive human-like qualities, such as motivation, in nonhuman entities (White \& Remington, 2018). Research also specifically showed increased social behaviors of autistic individuals towards toy objects (Boyd et al., 2007). The present work thus examined the relationship between autism and the mind attribution to toy objects.

\section{Selves as targets}

Another entity on which the current work focuses is the self. People in the normal population naturally judge themselves as possessing both agentic and experiential mental capacities (Wegner \& Gray, 2016), which originates from the direct experiences of their own mind. Individuals are usually aware of the mental states of themselves, which allows them to infer their own mind (Epley \& Waytz, 2010). Direct experiences of their own desires, beliefs, intentions, thoughts, and feelings, all bespeak the capacities for those psychological activities. Autistic individuals have, however, been found to have a diminished sense of psychological self (Williams, 2010). They tend to confuse their own intentional and unintentional actions, suggesting a limited recognition of their own intentions (Williams \& Happé, 2010). They also have difficulty understanding their own beliefs, which was even more severe than their difficulty understanding the beliefs of others (Williams \& Happé, 2009). With limited awareness of their own mental activities, autistic individuals are possibly less likely to perceive the mental capacities of themselves. They have even been reported to view themselves as less human (e.g., Prince-Hughes, 2004).

\section{Research overview}

In two studies, the relation between autism and mind perception of various types of entities was examined in normal adult samples. The studies were aimed at replicating and extending existing findings in the literature (Gray, Adrianna et al., 2011; Tharp et al., 2017). It was predicted that autism would negatively correlate with perception of human mind, and of agentic mental capacity in particular. The current work, furthermore, extended previous work to investigate the relation between autism and the perception of the mind of more types of entities, particularly toy objects (Studies 1 and 2) and the selves (Study 2).

\section{STUDY 1}

\section{Method}

\section{Participants}

Participants were 190 citizens of the United States recruited from Amazon's Mechanical Turk (MTurk). One participant provided an invalid MTurk ID and seven failed at least one of the two embedded attention checks. They were excluded from the analyses, leaving a final sample of 182 participants (91 females). Regarding the age of the participants in the final sample, $10 \%$ was 18 to $24,39 \%$ was 25 to $34,28 \%$ was 35 to $44,11 \%$ was 45 to 54 , and $12 \%$ was above 54 .

\section{Materials and procedure}

\section{Target entities}

Ten targets were used, including the two new targets, a toy teddy and a toy doll. All entities are listed in Table 1 . The presentation order of all the entities was randomized.

\section{Perceived capacity for experience and agency}

Participants rated all entities on perceived capacity for experience using three items (i.e., fear, pleasure, and hunger) and perceived capacity for agency using another three items (i.e., memory, self-control, and morality). These ratings were made on 11 -point scales $(0=$ not at all capable $; 10=$ fully capable). The reliabilities of both the experience ratings and the agency ratings for each entity were moderate to high (the $\alpha$ of experience ratings ranged from .76 to .93; the $\alpha$ of agency ratings ranged from .68 to .98).

\section{Autism-spectrum Quotient scale (AQ)}

Participants completed the Autism-spectrum Quotient scale (AQ), a self-report measure of autistic traits (Baron-Cohen et al., 2001). The AQ consists of 50 items, divided over five 
Table 1. Descriptive statistics for mind perception ratings and correlations between mind perception and the Autism-spectrum Quotient

\begin{tabular}{|c|c|c|c|c|c|c|c|}
\hline \multirow[b]{2}{*}{ Target } & \multirow[b]{2}{*}{$\begin{array}{l}\text { Mind Perception } \\
\text { Dimension }\end{array}$} & \multicolumn{3}{|c|}{ Study 1} & \multicolumn{3}{|c|}{ Study 2} \\
\hline & & $M$ & $S D$ & $\begin{array}{c}\text { Correlation } \\
\text { with AQ }\end{array}$ & $M$ & $S D$ & $\begin{array}{c}\text { Correlation } \\
\text { with AQ }\end{array}$ \\
\hline \multirow{2}{*}{ Male adult } & Agency & 8.39 & 2.13 & -0.11 & 8.69 & 1.93 & $-0.29 * *$ \\
\hline & Experience & 8.62 & 2.23 & -0.09 & 9.01 & 1.81 & $-0.23 * *$ \\
\hline \multirow{2}{*}{ Female adult } & Agency & 8.44 & 2.14 & -0.09 & 8.75 & 1.82 & $-0.33 * *$ \\
\hline & Experience & 8.53 & 2.35 & -0.08 & 9.11 & 1.77 & $-0.23 * *$ \\
\hline \multirow{2}{*}{ Dog } & Agency & 5.03 & 2.26 & -0.14 & 5.37 & 20.6 & 0.03 \\
\hline & Experience & 8.31 & 2.26 & -0.11 & 8.89 & 1.77 & $-0.19 *$ \\
\hline \multirow{2}{*}{ God } & Agency & 6.94 & 3.97 & 0.01 & 6.37 & 4.19 & -0.13 \\
\hline & Experience & 4.44 & 3.67 & 0.08 & 3.89 & 3.66 & -0.07 \\
\hline \multirow{2}{*}{ Infant } & Agency & 2.58 & 2.29 & 0.03 & 2.80 & 2.44 & 0.06 \\
\hline & Experience & 7.88 & 2.43 & -0.13 & 8.47 & 1.97 & $-0.19^{*}$ \\
\hline \multirow{2}{*}{ Robot } & Agency & 4.04 & 3.04 & -0.002 & 4.30 & 2.87 & -0.02 \\
\hline & Experience & 0.98 & 1.88 & 0.11 & 0.85 & 1.80 & $0.17 *$ \\
\hline \multirow{2}{*}{ Superman } & Agency & 5.40 & 4.34 & -0.04 & 6.67 & 4.08 & -0.004 \\
\hline & Experience & 4.26 & 3.88 & -0.02 & 5.61 & 3.94 & -0.04 \\
\hline \multirow{2}{*}{ Tree } & Agency & 1.21 & 2.26 & 0.03 & 1.11 & 2.32 & 0.15 \\
\hline & Experience & 1.65 & 2.55 & -0.05 & 1.61 & 2.58 & 0.07 \\
\hline \multirow{2}{*}{ Toy teddy } & Agency & 0.70 & 1.87 & $0.19^{*}$ & 0.73 & 1.95 & $0.20 *$ \\
\hline & Experience & 0.60 & 1.71 & $0.15^{*}$ & 0.66 & 1.86 & $0.16^{*}$ \\
\hline \multirow{2}{*}{ Toy doll } & Agency & 0.66 & 1.87 & $0.19 *$ & 0.58 & 1.68 & $0.24 * *$ \\
\hline & Experience & 0.48 & 1.41 & $0.20 *$ & 0.52 & 1.64 & $0.21 * *$ \\
\hline \multirow{2}{*}{ Self } & Agency & - & - & - & 8.78 & 1.81 & $-0.30 * *$ \\
\hline & Experience & - & - & - & 9.12 & 1.75 & $-0.24 * *$ \\
\hline
\end{tabular}

Note: Asterisks indicate significance of path coefficients $\left({ }^{*} p<.05, * * p<.01\right)$.

subscales, each containing 10 items, namely social skills (e.g., I find it hard to make new friends), attention switching (e.g., It does not upset me if my daily routine is disturbed), attention to detail (e.g., I tend to notice details that others do not), communication (e.g., I know how to tell if someone listening to me is getting bored), and imagination (e.g., If I try to imagine something, I find it very easy to create a picture in my mind). A 4-point scale was used ( $0=$ definitely disagree; 3 = definitely agree). The reliability of the total AQ was high $(\alpha=.83)$ and the reliabilities for the subscales were moderate to high (social skills, $\alpha=.84$; attention switching, $\alpha=.73$; attention to details, $\alpha=.74$; imagination, $\alpha=.66$; communication skills, $\alpha=.65$ ).

The order of the mind perception rating task and the AQ was counterbalanced. Finally, participants answered two attention check questions and a battery of other measures irrelevant to the present research questions.

\section{Results and discussion}

Descriptive statistics for the mind perception ratings and zero-order correlations between mind perception and AQ scores are displayed in the Study 1 columns of Table 1 . With the AQ scale allowing a total score from 0 to 150 , the mean of participants' AQ scores was $66.01(S D=14.03)$. Positive significant correlations between the AQ and mind perception of the two toy objects were found, for both the agency and the experience dimensions (toy teddy experience, $p=$ .04 ; toy teddy agency, $p=.01$; toy doll experience, $p=.01$; toy doll agency, $p=.01)$. There were no other statistically meaningful associations between AQ scores and mind perception. As in past work, the perception of the minds of other nonhuman entities was unrelated to AQ scores. Sur- prisingly, however, there were also no statistically significant associations between the AQ and the perception of adult minds ( $r$ ranged from -.11 to -.08). Overall, these results did not successfully replicate past findings. Nevertheless, note that the overall pattern of mind perception aligned with previous findings (e.g., Gray et al., 2007) and the rankorder of the mean ratings are shown in the Appendix.

\section{STUDY 2}

Study 1 had extended prior work on autism and mind perception as far as targets are concerned, and significant associations were found between autism and increased perception of the mental capacities in toy objects. Study 1, however, failed to replicate the negative associations that were found in past studies between autism and the perception of human agency. Study 2 was conducted to further ascertain the relationship of autism with mind perception of human entities and toy entities. Study 2 also investigated the relationship between autism and mind perception of another entity-the self.

\section{Method}

\section{Participants}

Participants were 171 American MTurk workers. Two participants provided an invalid MTurk ID and six failed at least one of the two embedded attention checks. These participants were excluded from the analysis, thus leaving a final sample of 163 participants ( 89 females). Of these participants, $15 \%$ was 18 to 24 years of age, $38 \%$ was 25 to 34 , 
$17 \%$ was 35 to $44,16 \%$ was 45 to 54 , and $14 \%$ was above 54 years of age. Most of the participants $(78 \%)$ were White/Caucasian. A tenth was Asian/Asian American, 7\% was Hispanic/Latino, and 4\% was African American.

\section{Materials and procedure}

Participants completed the mind perception questions and the AQ in an online questionnaire. They answered the same mind perceptions questions as in Study 1 on the ten targets and on the additional Self target. The presentation order of the 11 entities was randomized. The reliability of the experience ratings for each entity was moderate to high ( $\alpha$ ranged from .73 to .98 ), as well as the reliability of the agency ratings for each entity ( $\alpha$ ranged from .55 to .98). The reliability of the total AQ was high $(\alpha=.81)$. The reliabilities for the subscales were moderate (social skills, $\alpha=.80$; attention switching, $\alpha=.60$; attention to details, $\alpha=.61$; imagination, $\alpha=.69$; communication skills, $\alpha=.57$ ). The mind perception task and the AQ were counterbalanced. Participants also answered two attention checks and other measures unrelated to the present research questions.

\section{Results and discussion}

Descriptive statistics for the mind perception ratings and the correlations between mind perception and the AQ are displayed in the Study 2 column in Table 1 . The mean AQ score was $65.05(S D=15.36)$. In agreement with the results of study $1, \mathrm{AQ}$ and mind perception of the two toy objects were significantly positively correlated, for both agency and experience (toy teddy experience, $p=.04$; toy teddy agency, $p=.01$; toy doll experience, $p=.01$; toy doll agency, $p=$ $.002)$. Moreover, AQ was found to be significantly negatively correlated with mind perception of the self as a target. Participants who scored higher on AQ tended to perceive themselves as having less mental capacity for both experience $(p=.002)$ and agency $(p<.001)$. These latter findings extended prior work by showing that autism was not only negatively associated with the perceptions of other adults' minds but also of the participants' own minds, on both the agency and experience dimensions. In addition, significant negative correlations were found between AQ and the perceptions of adult minds (female adult experience, $p<.001$; female adult agency, $p<.001$; male adult experience, $p=$ .003 ; male adult agency, $p<.001$ ), which contrasted to the findings in Study 1, where no significant correlations were found. Some other small-sized, yet significant, correlations were found between autism and perceptions of the experience of human infants, dogs, and robots. Overall, Study 2 showed a positive relationship between autism and mind perception of toy objects and a negative relationship between autism and the perception of adult minds - both the minds of others and of selves.

\section{Mini meta-analysis}

Because of some inconsistences in the findings from Study 1 to Study 2, a mini meta-analysis using fixed effects was conducted to provide improved estimates of the effect sizes of the correlations between autism and mind perception
(Goh et al., 2016). The Stouffer's $Z$ test was also conducted to generate a summary $p$ value for each correlation. Across the two studies, AQ was negatively associated with both perceived experience $(r=-.16, Z=-2.91, p<.001)$ and agency $(r=-.20, Z=-3.67, p<.001)$ of a male adult, as well as with both perceived experience $(r=-.15, Z=-2.81, p=$ $.002)$ and agency $(r=-.21, Z=-3.86, p<.001)$ of a female adult. The effect sizes of the associations between autism and adult experience perception were small, while the effect sizes of the associations between autism and adult agency perception were small to medium. AQ, moreover, was positively associated with perceived experience $(r=.16, Z=$ $2.87, p=.004)$ and agency $(r=.20, Z=3.63, p<.001)$ of a toy teddy, as well as with perceived experience $(r=.21, Z=$ $3.82, p<.001)$ and agency $(r=.21, Z=4.00, p<.001)$ of a toy doll. The effect sizes were small to medium. Overall, a positive relation between autism and the perception of the mind of toy objects and a negative one between autism and the perception of human mind were supported.

\section{GENERAL DISCUSSION}

Autism does not only exist in the clinical population but also in the normal population (e.g., Baron-Cohen et al., 2001). Past work has shown that, in the normal population, being more autistic can influence mind perception (Gray, Adrianna et al., 2011; Tharp et al., 2017). In particular, a negative link between autism and the perception of human mind on the agency dimension has been found.

The current work produced a partial replication of past research, as well as some novel findings. In two normal adult samples, small- to medium-sized negative associations were found between autism and the ascription of mental capacities to other adults. Unlike past work, autism was found to be associated with both decreased perception of human agency and decreased perception of experience. Autism was, moreover, associated with decreased perception of adult minds of others and of the mind of oneself, such that more autistic individuals tended to perceive less mental capacity in themselves. Autism was also positively associated with mind attribution to toy objects, both to toy teddies and to toy dolls.

The negative associations found between autism and the perception of human mind suggest a disposition associated with the autism spectrum to dehumanize others. Past research has only shown associations of autism with decreased perceptions of human agency (Gray, Adrianna et al., 2011). This implies that autistic individuals would tend to see less uniquely human characteristics in other human adults, thus viewing them as more similar to nonhuman animals (Haslam, 2006). The current work, however, suggested that autism additionally had associations with reduced perception of experiential mental capacity in human adults, implying that autism was also negatively related to the attribution of affective human nature characteristics to others, thereby contributing to the views of other people merely as objects (Haslam, 2006). The current findings, furthermore, showed a negative relationship between autism and mind attribution to oneself, suggesting a possible relationship between autism and self-dehumanization (Kouchaki et al., 2018). 
The current work also showed associations between autism and increased mind perception of toy objects. This opened up the possibilities of autistic individuals using toys to fulfill their social needs. Autistic individuals tend to have little social interaction with others (e.g., Chevallier et al., 2012), but they may choose other entities to interact with, particularly those that are highly predictable. Toys are one possible choice. Indeed, previous work has documented some social interactions of autistic children toward toy objects (Boyd et al., 2007). Thus, it is possible that autistic individuals can attribute more mental capacity to toy objects, rendering them a possible source of social connection. The direct relationship between social interactions with toy objects and mind attribution to them, however, needs further research.

The study of the relationship between autism and mind perception is of crucial importance because of the strong implications of mind perception for daily judgments and behaviors. Mind perception is important to morality, with experiential mental capacities predicting moral respect and agentic ones predicting moral responsibility (Gray et al., 2007; K. Gray et al., 2012; Waytz et al., 2010b). With autism being related to reduced mind attribution to other adults, more autistic individuals may see fewer moral rights and responsibilities in others, which could legitimize doing harm to them (Waytz et al., 2010a). The positive associations between autism and mind perception of toy objects, by contrast, suggested that autistic individuals may grant more moral rights to toy objects and offer them more moral care (Waytz et al., 2010a). Furthermore, the associations found between autism and reduced mind attribution to oneself suggested the possibility that more autistic individuals assign less blame to themselves when they act unethically, thereby increasing their chances of doing so (Kouchaki et al., 2018). Future research may substantiate these possibilities.

There are several points regarding this research that are noteworthy to the readers. The first is about the correlational nature of the findings. The present research only showcased the relationship between autism and mind perception. The direction of causality cannot be confirmed as both mind perception and autism were only measured in each study. Another noteworthy point pertains to the differentiation between autism found in the normal population and the clinical diagnoses of the ASC. Some past research focused on mind perception of individuals diagnosed with ASC (e.g., Akechi et al., 2018) and found that they perceived mental capacities along the same dimensions - agency and experience-as their normal counterparts, despite slight differences in the quantity of mental capacities attributed to some entities. Other research (Gray, Adrianna et al., 2011; Tharp et al., 2017) and the present work focused on how autism found in normal adults is related to mind perception. The present research added to the literature that autism in the normal population is related to reduced mind perception of the self and increased mind perception of toy objects.

\section{Conclusion}

Autism and mind perception are interrelated. Prior work has shown that, among normal adults, autism was associated with reduced perception of agentic mental capacities of others. The present research provided support to the previous findings and added that autism was also significantly associated with decreased mind perception of oneself and increased mind perception of toy objects. The current findings have shed light on how autism in the normal population may influence people's views of the minds of various entities. These perceptions have implications for the potential effects of autism on the treatment of various types of entities.

\section{ACCOUNTS AND ACKNOWLEDMENTS}

This work was partially supported by the Department of Psychology of the Chinese University of Hong Kong.

This article is based in part on the master's thesis completed by Rachel Hoi Yan Au.

\section{Ethical considerations}

The studies were granted ethical approval by the Survey and Behavioural Research Ethics Committee at the Chinese University of Hong Kong.

\section{Conflict of interest}

The author declared no potential conflicts of interest with respect to the research, authorship, and/or publication of this article.

\section{Data}

The data of both studies are publicly available on the Open Science Framework (OSF) website:

(https://osf.io/pkduy/?view_only=a90368f7540e42118177 0b60f54ea0bd).

\section{REFERENCES}

Akechi, H., Kikuchi, Y., Tojo, Y., Hakarino, K., \& Hasegawa, T. (2018). Mind perception and moral judgment in autism. Autism Research, 11, 1239-1244. https://doi.org/10.1002/aur.1970

American Psychiatric Association. (2013). Diagnostic and statistical manual of mental disorders (5th ed.). Arlington, VA: American Psychiatric Association.

Atherton, G., \& Cross, L. (2018). Seeing more than human: autism and anthropomorphic theory of mind. Frontiers in Psychology, 9, 528. https://doi.org/10.3389/fpsyg.2018.00528

Baron-Cohen, S., Wheelwright, S., Skinner, R., Martin, J., \& Clubley, E. (2001). The autism-spectrum quotient (AQ): Evidence from Asperger syndrome/high-functioning autism, males and females, scientists and mathematicians. Journal of Autism and Developmental Disorders, 31, 5-17.

https://doi.org/10.1023/A:1005653411471

Baron-Cohen, S. (2002). The extreme male brain theory of autism. Trends in Cognitive Sciences, 6, 248-254. https://doi.org/10.1016/S1364-6613(02)01904-6

Baron-Cohen, S., Richler, J., Bisarya, D., Gurunathan, N., \& Wheelwright, S. (2003). The systemizing quotient: An investigation of adults with Asperger syndrome or high-functioning autism, and normal sex differences. Philosophical Transactions of the Royal Society of London. Series B: Biological Sciences, 358, 361-374. https://doi.org/10.1098/rstb.2002.1206 
Boyd, B. A., Conroy, M. A., Mancil, G. R., Nakao, T., \& Alter, P. J. (2007). Effects of circumscribed interests on the social behaviors of children with autism spectrum disorders. Journal of Autism and Developmental Disorders, 37, 1550-1561.

https://doi.org/10.1007/s10803-006-0286-8

Chevallier, C., Kohls, G., Troiani, V., Brodkin, E. S., \& Schultz, R. T. (2012). The social motivation theory of autism. Trends in Cognitive Sciences, 16, 231-239. https://doi.org/10.1016/j.tics.2012.02.007

Constantino, J. N., \& Todd, R. D. (2003). Autistic traits in the general population: A twin study. Archives of General Psychiatry, 60, 524-530. https://doi.org/10.1001/archpsyc.60.5.524

Cooley, E., Payne, B. K., Cipolli III, W., Cameron, C. D., Berger, A., \& Gray, K. (2017). The paradox of group mind: "People in a group" have more mind than "a group of people". Journal of Experimental Psychology: General, 146, 691.

https://doi.org/10.1037/xge0000293

Doyle, C. M., \& Gray, K. (2020). How people perceive the minds of the dead: The importance of consciousness at the moment of death. Cognition, 202, 104308. https://doi.org/10.1016/j.cognition.2020.104308

Epley, N., Akalis, S., Waytz, A., \& Cacioppo, J. T. (2008). Creating social connection through inferential reproduction: Loneliness and perceived agency in gadgets, gods, and greyhounds. Psychological Science, 19, 114-120. https://doi.org/10.1111/j.1467-9280.2008.02056.x

Epley, N., \& Waytz, A. (2010). Mind perception. In S. T. Fiske, D. T. Gilbert, \& G. Lindzey (Eds.), Handbook of social psychology (pp. 498-541). John Wiley \& Sons, Inc. https://doi.org/10.1002/9780470561119.socpsy001014

Epley, N., Waytz, A., Akalis, S., \& Cacioppo, J. T. (2008). When we need a human: Motivational determinants of anthropomorphism. Social Cognition, 26, 143-155. https://doi.org/10.1521/soco.2008.26.2.143

Ford, T. C., Apputhurai, P., Meyer, D., \& Crewther, D. P. (2017). Confirmatory factor analysis of autism and schizophrenia spectrum traits. Personality and Individual Differences, 110, 80-84. https://doi.org/10.1016/j.paid.2017.01.033

Gardner, W. L., \& Knowles, M. L. (2008). Love makes you real: Favorite television characters are perceived as "real" in a social facilitation paradigm. Social Cognition, 26, 156-168. https://doi.org/10.1521/soco.2008.26.2.156

Goh, J. X., Hall, J. A., \& Rosenthal, R. (2016). Mini meta-analysis of your own studies: Some arguments on why and a primer on how. Social and Personality Psychology Compass, 10, 535-549. https://doi.org/10.1111/spc3.12267

Goris, J., Brass, M., Cambier, C., Delplanque, J., Wiersema, J. R., $\&$ Braem, S. (2020). The relation between preference for predictability and autistic traits. Autism Research, 13, 1144-1154. https://doi.org/10.1002/aur.2244

Gray, H. M., Gray, K., \& Wegner, D. M. (2007). Dimensions of mind perception. Science, 315, 619-619. https://doi.org/10.1126/science.1134475

Gray, K., Adrianna, C., Jenkins, A. C., Andrea, S., Heberlein, A. S., \& Wegner, D. M. (2011). Distortions of mind perception in psychopathology. Proceedings of the National Academy of Sciences, 108, 477-479. https://doi.org/10.1073/pnas.1015493108

Gray, K., Knobe, J., Sheskin, M., Bloom, P., \& Barrett, L. F. (2011). More than a body: Mind perception and the nature of objectification. Journal of Personality and Social Psychology, 101, 1207. https://doi.org/10.1037/a0025883

Gray, K., Young, L., \& Waytz, A. (2012). Mind perception is the essence of morality. Psychological Inquiry, 23, 101-124. https://doi.org/10.1080/1047840X.2012.651387

Haslam, N. (2006). Dehumanization: An integrative review. Personality and Social Psychology Review, 10, 252-264.

https://doi.org/10.1207/s15327957pspr1003_4
Jobe, L. E., \& White, S. W. (2007). Loneliness, social relationships, and a broader autism phenotype in college students. Personality and Individual Differences, 42, 1479-1489. https://doi.org/10.1016/j.paid.2006.10.021

John, O. P., \& Srivastava, S. (1999). The Big Five trait taxonomy: History, measurement, and theoretical perspectives. In S.T. Fiske, D.T. Gilbert, \& G. Lindzey (Eds.), Handbook of personality: Theory and research, 2, 102-138.

Kouchaki, M., Dobson, K. S., Waytz, A., \& Kteily, N. S. (2018). The link between self-dehumanization and immoral behavior. Psychological Science, 29, 1234-1246. https://doi.org/10.1177/0956797618760784

Kozak, M. N., Marsh, A. A., \& Wegner, D. M. (2006). What do I think you're doing? Action identification and mind attribution. Journal of Personality and Social Psychology, 90, 543. https://doi.org/10.1037/0022-3514.90.4.543

Malle, B. F. (2019). How many dimensions of mind perception really are there? In E. K. Goel, C. M. Seifert, \& C. Freksa (Eds.), Proceedings of the 41st Annual Meeting of the Cognitive Science Society (pp. 2268-2274). Montreal, Canada: Cognitive Science Society.

Mazurek, M. O. (2014). Loneliness, friendship, and well-being in adults with autism spectrum disorders. Autism, 18, 223-232. https://doi.org/10.1177/1362361312474121

Michaud, F., \& Théberge-Turmel, C. (2002). Mobile robotic toys and autism. In K. Dautenhahn, A. Bond, L. Cañamero \& B. Edmonds (Eds.), Socially Intelligent Agents (pp. 125-132). Springer, Boston, MA.

Prince-Hughes, D. (2004). Songs of the gorilla nation: My journey through autism. Crown.

Tharp, M., Holtzman, N. S., \& Eadeh, F. R. (2017). Mind Perception and Individual Differences: A Replication and Extension. Basic and Applied Social Psychology, 39, 68-73. https://doi.org/10.1080/01973533.2016.1256287

Van de Cruys, S., Evers, K., Van der Hallen, R., Van Eylen, L., Boets, B., de-Wit, L., \& Wagemans, J. (2014). Precise minds in uncertain worlds: predictive coding in autism. Psychological Review, 121, 649. https://doi.org/10.1037/a0037665

Waytz, A., Epley, N., \& Cacioppo, J. T. (2010a). Social cognition unbound: Insights into anthropomorphism and dehumanization. Current Directions in Psychological Science, 19, 58-62. https://doi.org/10.1177/0963721409359302

Waytz, A., Gray, K., Epley, N., \& Wegner, D. M. (2010b). Causes and consequences of mind perception. Trends in Cognitive Sciences, 14, 383-388. https://doi.org/10.1016/j.tics.2010.05.006

Waytz, A., Morewedge, C. K., Epley, N., Monteleone, G., Gao, J. H., \& Cacioppo, J. T. (2010c). Making sense by making sentient: effectance motivation increases anthropomorphism. Journal of Personality and Social Psychology, 99, 410. https://doi.org/10.1037/a0020240

Wegner, D. M., \& Gray, K. (2016). The Mind Club: Who Thinks, What Feels, and Why It Matters. New York, NY: Viking.

White, R. C., \& Remington, A. (2019). Object personification in autism: This paper will be very sad if you don't read it. Autism, 23, 1042-1045. https://doi.org/10.1177/1362361318793408

Williams, D. (2010). Theory of own mind in autism: Evidence of a specific deficit in self-awareness?. Autism, 14, 474-494. https://doi.org/10.1177/1362361310366314

Williams, D. M., \& Happé, F. (2009). What did I say? Versus what did I think? Attributing false beliefs to self amongst children with and without autism. Journal of Autism and Developmental Disorders, 39, 865-873. https://doi.org/10.1007/s10803-009-0695-6

Williams, D., \& Happé, F. (2010). Representing intentions in self and other: Studies of autism and typical development. Developmental Science, 13, 307-319.

https://doi.org/10.1111/j.1467-7687.2009.00885.x

Received November 10, 2020 Accepted January 16, 2021 


\section{APPENDIX}

Examining the rank-order of the mean ratings of mind perception in the two studies, it was found that participants had the general patterns of mind perception documented in previous work (Gray et al., 2007; Wegner \& Gray, 2016). Human entities were perceived as having the most agency, followed by supernatural agents, then by human infants and nonhuman animals, then by nature, then by toy objects. Human entities were perceived as having the most experience, followed by human infants and nonhuman animals, then by supernatural agents, then by nature, then by toy objects.

Rank-order of the means for mind perception of the targets in Study 1 and Study 2

\begin{tabular}{|c|c|c|c|}
\hline \multicolumn{2}{|c|}{$\begin{array}{c}\text { Study } 1 \\
\text { Mind perception dimensions }\end{array}$} & \multicolumn{2}{|c|}{$\begin{array}{c}\text { Study } 2 \\
\text { Mind perception dimensions }\end{array}$} \\
\hline Agency & Experience & Agency & Experience \\
\hline Female adult & Male adult & Self & Self \\
\hline Male adult & Female adult & Female adult & Female adult \\
\hline God & Dog & Male adult & Male adult \\
\hline Superman & Infant & Superman & Dog \\
\hline Dog & God & God & Infant \\
\hline Robot & Superman & Dog & Superman \\
\hline Infant & Tree & Robot & God \\
\hline Tree & Robot & Infant & Tree \\
\hline Toy teddy & Toy teddy & Tree & Robot \\
\hline Toy doll & Toy doll & Toy teddy & Toy teddy \\
\hline- & - & Toy doll & Toy doll \\
\hline
\end{tabular}

